\title{
Brief Fear of Negative Evaluation Scale-II and Brief Fear of Negative Evaluation Scale-Straightforward Items: psychometric properties of the Turkish versions
}

\author{
Battal Gokturk Gok ${ }^{\oplus}$, Ozden Yalcinkaya-Alkar ${ }^{\circledR}$ \\ ${ }^{1}$ Hacettepe University, Department of Psychology, Ankara - Turkey \\ ${ }^{2}$ Ankara Yildirim Beyazit University, Department of Psychology, Ankara - Turkey
}

\begin{abstract}
Objective: The present study aimed at examining the psychometric properties of the Brief Fear of Negative Evaluation Scale-II (BFNE-II) and the BFNE-Straightforward Items (BFNE-S) in a non-clinical Turkish sample.

Method: The sample consisted of a total of 243 undergraduate students. Confirmatory Factor Analysis (CFA) was done for the BFNE-II and the BFNE-S to confirm the proposed unitary model; internal consistency coefficient was calculated, and reliability coefficients were determined by using the split-half method. For concurrent validity, the Fear of Positive Evaluation Scale, the Liebowitz Social Anxiety Scale, and the Rosenberg Self-Esteem Scale were used.

Results: As a result of CFA, the BFNE-II and the BFNE-S showed a good fit to the unitary model as in the original form. The Cronbach's Alpha reliability coefficient of the BFNE-II was found to be 0.92 and the reliability coefficient determined by the split-half method was 0.94; the reliability coefficients of the BFNE-S were 0.90 for the Cronbach's Alpha and 0.91 for the splithalf method. The analysis also revealed that BFNE-II and BFNE-S have good concurrent validity with the measures of the fear of positive evaluation, self-esteem and social anxiety.

Conclusion: These findings support the use of the brief fear of negative evaluation revisions (BFNE-II and BFNE-S) in Turkish culture as measures of fear of negative evaluation in research. The implications of the research were discussed in light of the related literature.
\end{abstract}

Keywords: Brief Fear of Negative Evaluation Scale-Il, fear of negative evaluation, social anxiety, psychometric properties

\section{INTRODUCTION}

The main characteristic of social anxiety disorder (SAD) is defined in DSM-5 as having an obvious or intense fear or anxiety in social situations where the individual is likely to be evaluated by others (1). Fear of negative evaluation is also seen as one of the basic characteristics of SAD in cognitive-behavioral models $(2,3)$. Similarly,
Beck et al. (4) argued that the main fear in social anxiety is the fear of being negatively evaluated by another person or persons and distinguishes social anxiety from other anxiety types. These models are consistent with the empirical findings in which individuals with high social anxiety levels report negative mental representations of their appearance and behavior, particularly in anxiety causing social situations, and

How to cite this article: Gok BG, Yalcinkaya-Alkar O. Brief Fear of Negative Evaluation Scale-Il and Brief Fear of Negative Evaluation ScaleStraightforward Items: psychometric properties of the Turkish versions. Dusunen Adam The Journal of Psychiatry and Neurological Sciences 2020;33:410-420.

Correspondence: Battal Gokturk Gok, Hacettepe University, Department of Psychology, 06800 Beytepe, Ankara - Turkey 
they view these social situations from an observational perspective (through someone else's eyes) (5-7).

Fear of negative evaluation is associated with the fear of being evaluated as negative or bad while participating in a social situation or expecting a social situation, while social anxiety is associated with affective reactions to these situations (8). In particular, the fear of negative evaluation means anxiety, annoyance, and fear resulting from humiliating, critical, or unfriendly judgment by others, and from thoughts and beliefs about being disgraced or ridiculed.

Fear of Negative Evaluation Scale (FNE) was first developed by Watson and Friend (9). The FNE consists of a total of 30 true-false items with 17 straightforwardly-worded (directly scored) and 13 reverse-worded items (reverse scored). Individuals with high scores in the FNE are more likely to make negative generalizations about uncertain social situations, and also tend to catastrophize mildly adverse social situations compared to individuals with low scores (10). The Turkish validity and reliability study of the scale was carried out by Erkan et al. (11) in the adolescent sample. The original and Turkish forms of FNE have adequate psychometric properties.

Although it has been possible to use FNE widely in researches, the first version of the short form of the scale was created by Leary (12), as the scale is long and the two-dimensional response options sometimes interfere with its benefits. The Brief Fear of Negative Evaluation Scale (BFNE) is the most commonly used measurement tool to determine the degree of anxiety about the possibility of humiliation, critical or hostile judgment, and disgrace by others (12). The BFNE consists of 12 items, 11 of which are exactly the same as FNE. Eight of these items are straightforwardly-worded (directly scored) and 4 are reverse-worded (reverse scored). In addition, the items have a five-point Likert-type scoring system instead of a true-false format with a twodimensional response option. Leary (12) stated that the psychometric properties of BFNE were almost identical to the long form of FNE, the original version of the scale, (9), and the total scores of the scales were highly correlated $(\mathrm{r}=0.96)$. Examining the Cronbach's alpha value in the BFNE sample of undergraduate students, a high internal consistency $(\alpha=0.90)$ and a 4 -week testretest reliability $(\mathrm{r}=0.75)$ were found (12). In the study conducted by Weeks et al. (8), BFNE showed excellent internal consistency in the clinical sample $(\alpha=0.90)$, while the internal consistency in the non-clinical control group was minimal $(\alpha=0.67)$. However, a good internal consistency coefficient was found for the entire sample $(\alpha=0.81)$. The Turkish adaptation of BFNE was first carried out by Koydemir and Demir (13). Bilge and Kelecioglu (14) examined the psychometric properties of the Turkish form in a high school sample. In the following years, a validity and reliability study on the first version of the short form was conducted by Cetin et al. (15).

In the first studies conducted when the BFNE was put into use, it was assumed that the factor structure of the scale has a one-dimensional structure $(10,12,16)$. In the next period, Rodebaugh et al. (17) applied confirmatory factor analysis (CFA) on FNE and BFNE to a sample of 1049 university students. As a result of the analysis, it was revealed that the BFNE, which has a Likert-type rating system, was a more sensitive measurement tool than the FNE, which has a binary evaluation system in providing information; and it could determine the level of violence of the latent structure (fear of negative evaluation) in a wider range. The null hypothesis results indicated that the binary evaluation system of the FNE could not distinguish the high levels of fear resulting in a ceiling effect. In other words, there was little room for variation as all participants score higher. These results appeared to be consistent with previous studies $(16,18)$ that while FNE successfully distinguished individuals with anxiety disorders from individuals without anxiety disorders, it could not consistently identify individuals with panic disorder, agoraphobia, and SADs. In addition, the assumed unitary factor structure for FNE and BFNE showed a better fit compared to a multi-factor model, and therefore the researchers concluded that it would not be appropriate to use the unitary model (18). As a result of the CFA, a two-factor structure that includes items that are straightforwardly worded such as "I am usually worried about what kind of impression I make" and items that are reversely worded such as "Other people's opinions of me do not bother me". Another problem addressed in the studies is the items worded reversely. Although the use of such items is sometimes recommended to detect response errors, it is considered that these items tend to confuse the participants' minds (17). As a result of the analysis of the data, the researchers suggested using only straightforwardly-worded items $(8,17)$. In line with the recommendations in the literature, the updated and reverse-scored items were reviewed and the BFNE-II was developed, in which only the straightforwardly worded items were used (19). As a result of the CFA performed on the BFNE-II, a unitary model was generated with no loss of sensitivity due to the revised items. 
As can be understood from the literature, scale development and adaptation studies have been carried out extensively on the fear of negative evaluation. In line with long-standing studies, the scale continues to be updated and compared with previous versions. Adaptation studies of the old and current versions of the scale continue to be carried out in different languages and cultures such as Spanish (20), Iranian (21), and Chinese (22). Within the scope of the adaptation study, it is planned to perform the validity and reliability study of BFNE-II (19). Furthermore, it was aimed to examine the psychometric properties of the scale the BFNEStraightforward Items (BFNE-S), which was obtained by removing the revised 4 -items of the scale, namely the reversed items of the BFNE (items 2, 4, 7 and 10). In other words, it was aimed to adapt a more updated version of the scale to Turkish and to examine its validity and reliability in line with the increasing knowledge and suggestions about fear of negative evaluation.

\section{METHOD}

\section{Participants}

This research was approved by the Ankara Yildirim Beyazit University Ethics Committee. The sample of the study consists of 243 undergraduate students from Ankara Yildirim Beyazit University and Kirsehir Ahi Evran University. In the power analysis performed with $\mathrm{R}$, a sufficient sample size was calculated to perform CFA. In the analyzes, it was determined that 238 participants at the 0.05 RMSEA level should achieve 0.80 statistical power $(\mathrm{p}<0.05)$. In this study, the statistical power for BFNE-II with 243 participants was calculated as 0.81 . In addition, the sample size meets the ratio of at least 5 participants (5:1) to a maximum of 10 participants (10:1) per item, which is widely accepted in the relevant literature (23).

The inclusion criteria are being in the 18-45 age range, being a university student, willingness, and motivation to take part in the research. In addition, a written informed consent form must be signed to participate in the study. Problems related to Turkish (speaking, reading and writing) are included in the exclusion criteria. In the current sample, there are 183 female $(75.3 \%)$ and $60(24.7 \%)$ male participants who meet these conditions; the sample was taken from the non-clinical population. The average age of the participants between the ages of 18 and 26 is 21.14 $(\mathrm{SD}=1.61)$. There was no significant difference in age between males and females ( $\mathrm{t}[241]=-1.05, \mathrm{p}=0.29$ ). Regarding their departments, $65 \%$ of the participants are Turkish Language and Literature students, 14\% Geography students, $11.5 \%$ History students, followed by the Psychology department with 9.5\%. At this point, for additional notice, it should be mentioned that all participants in the study completely filled out the data collection tools. Researchers did not choose to remove any participant from the data set.

\section{Measures}

Brief Fear of Negative Evaluation Scale-II (BFNE-II): The BFNE-II, developed by Carleton et al. (19), is a revised version of the BFNE items developed by Leary (12) under the suggestions of the relevant literature. As a result of the revision of four items with reverse worded (item 2, item 4, item 7, and item 10), the BFNE-II consists of 12 items that are worded only straightforwardly (directly scored). The scale has a fivepoint Likert-type rating system (between $1=$ not at all characteristic of me and $5=$ entirely characteristic of me). Cronbach's alpha internal consistency coefficient of BFNE-II was determined as $\alpha=0.96$ (19) and $\alpha=0.97$ (24) in the Canadian sample.

Fear of Positive Evaluation Scale (FPES): It was developed by Weeks et al. (25) to measure the fear of positive evaluation, which is considered a possible feature of social anxiety. The scale consists of 10 items in total and has a ten-Likert-type rating system (between $0=$ not at all true and $9=$ very true). Two inversely evaluated items (item 5 and item 10) were included in the scale as filler in order to prevent response bias. These two items are removed from the total score calculation and the remaining 8 items are evaluated. The internal consistency coefficient of FPES in the general sample was reported as 0.80 , and the test-retest reliability coefficient performed after 5 weeks was 0.70 . As a result of CFA, the 8-item model was found to fit good. The validity and reliability study of the Turkish form of the scale was conducted by Doğan and Totan (26). In this study, the internal consistency coefficient of the scale was 0.73 , and the test-retest reliability coefficient was 0.75 . In the research, it is seen that the KMO coefficient is 0.79 .

Liebowitz Social Anxiety Scale (LSAS): The scale was developed by Liebowitz (27). Consisting of 24 items in total, 11 items are divided into two subscales that evaluate social interaction status and the other 13 items evaluate performance anxiety (28). The anxiety and avoidance severity experienced by the person in the last week are assessed separately for fear $(0=$ none, $1=$ mild, $2=$ moderate, $3=$ severe) and avoidance $(0=$ never, $1=$ occasionally, $2=$ often, $3=$ usually $)$ 
subscales. Cronbach's alpha coefficient of LSAS varies between 0.81 and 0.92 (28). The validity and reliability study of the LSAS Turkish form was conducted by Soykan et al. (29). The inter-rater reliability coefficient of the Turkish form was reported as 0.96 for the whole scale, 0.96 for the fear or anxiety subscale, and 0.95 for the avoidance subscale. Test-retest reliability was determined as 0.97 for all subscales and the total scale. Considering the internal consistency values of the scale, Cronbach's alpha coefficients were 0.96 for the fear or anxiety subscale, 0.95 for avoidance, and 0.98 for the whole scale.

Rosenberg Self-Esteem Scale (RSES): Developed by Rosenberg (30), the self-esteem subscale, which has a four-point Likert type rating system, consists of a total of 10 items, five of which are positive and five negative. The scoring of the scale is made by using the Guttman assessment technique. The scores that participants can obtain from the scale range from $0-6$. The score in the range of 0-1 indicates high, 2-4 points medium and 5-6 points indicate low self-esteem level. Accordingly, the high scores obtained from the scale correspond to low self-esteem levels. The Turkish validity and reliability study of RSES was carried out by Cuhadaroglu (31). The validity coefficient of the scale was found to be 0.71 . Test-retest reliability coefficients for the subscales ranged from 0.46 (feeling threatened in interpersonal relationships) to 0.89 (psychosomatic symptoms). The test-retest reliability coefficient for the self-esteem subscale was found to be 0.75 .

\section{Process}

After getting permission and support from Dr. Nicholas Carleton, one of the researchers who developed the original scale in English, via e-mail, the translation and adaptation process started. During the adaptation process of the scale, the criteria suggested by Hambleton and Putsala (32) were taken into consideration, and attention was paid to translate in accordance with the culture. First of all, the translators were chosen from among those who were fluent in both languages, familiar with the culture being studied and knew about the test and measurement structure. The scale was translated into Turkish by four research assistants specialized in social sciences, and a single form was created by selecting a translation for each item among the different translations by a group of 5 clinical psychology graduate students under the guidance of an expert associate professor. In the next stage, the scale was applied to 10 clinical psychology graduate students, and the language comprehensibility was assessed through clarity/comprehensibility questions and the answer alternatives were determined between 1 and 5 (not clear/not understandable and very clear/understandable at all). Then it was reviewed whether there was a score below 3 in the evaluation. As a result of the feedback, it was observed that all items of the agreed form of the scale were comprehensible.

The data collection process started following the approval of the Ankara Yildirim Beyazit University Ethics Committee. The data were collected personally by students studying in different departments. In the first stage, permission was obtained from the instructors to apply the questionnaires, then the volunteer students were asked to fill out the data collection set. It is worth noting here that students were not encouraged to participate in the study through bonus points, money or any other means. Those wishing to take part in the study were made to read the informed consent form, which includes general information about the study (such as the purpose of the study and by whom it was conducted), confidentiality, and volunteering requirements, and their approval was obtained. After their voluntary participation in the study was achieved, the participants filled out the demographic information form, the BFNE-II, which covers all the necessary items to measure the alternative item set of BFNE-S and other measurement tools. All data collected from the participants were obtained via pen filling forms.

\section{Statistical Analysis}

First, descriptive statistics were calculated over the data set; the mean, standard deviation, range, skewness, and kurtosis values were determined separately for all BFNE-II items (since it also includes BFNE-S items). DFA was conducted through AMOS 22 program to test whether BFNE-II and BFNE-S consist of a single-factor structure as in the original forms. In the calculation of statistical power R and for other analyzes, Statistical Package for Social Sciences 23.0 package program was used. For concurrent validity, the correlation coefficients of BFNE-II and BFNE-S with FPES, LSAS, and Pearson product-moments were calculated. For the reliability of the scales, Cronbach's alpha internal consistency coefficient and item-total correlations were calculated. For another reliability analysis, reliability coefficients were determined using the split-half method. Finally, independent groups t-test analysis was used to test the sensitivity of BFNE-II and BFNE-S to differences by gender. 


\section{RESULTS}

\section{Descriptive Statistics}

First of all, univariate kurtosis and skewness indices were calculated since failure to achieve normality may negatively affect multivariate data analyzes such as CFA. It was determined that all items and the total scale were within acceptable limits in terms of kurtosis and skewness values. These values meet the value criteria between -2.0 and +2.0 proposed by George and Mallery (33). In other words, none of these indexes were found outside of the range that was determined as extreme. The mean score for BFNE-II was found to be $28.27(\mathrm{SD}=11.05)$ and the scores obtained ranged from 12 to 48. On the other hand, in BFNE-S, these values were average $17.51(\mathrm{SD}=7.54)$ and the scores range from 8 to 40 . The descriptive statistics for the responses of the sample to the BFNE-II and BFNE-S items are shown in Table 1.

\section{Confirmatory Factor Analysis Findings}

It is known that the original form of BFNE-II consists of a single-factor structure $(19,24)$. Therefore, CFA was performed to test the single-factor structure in the original form of BFNE-II. The goodness of fit statistics obtained as a result of the CFA was taken as criteria to evaluate the validity of the original form of the scale in Turkish culture. While evaluating the CFA results, the following criteria were taken into account in terms of fit indexes: (1) Chi-square $\left(\chi^{2}\right)$ value should not be significant (34); (2) The $\chi^{2} / \mathrm{df}$ ratio should be less than 3
(35) for an acceptable fit or less than 2 for a good fit (36); (3) The Tucker Lewis Index (TLI) should be close to or exceed 0.95 (37); (4) The Comparative Fit Index (CFI) should be 0.95 or more for a good fit and 0.90 or more for an acceptable fit (37); (5) Root Mean Square Error of Approximation (RMSEA) should be less than 0.05 for a good fit, whereas less than 0.08 for an acceptable fit (38); (6) The Standardized Root Mean Square Residual (SRMR) should be 0.05 or less for a good fit and 0.10 or less for an acceptable fit (39). As the chi-square is sensitive to sampling (36), other fit index criteria will be emphasized in evaluations for a more precise and accurate assessment of the data set. As a result of the initial analysis performed with the maximum likelihood estimation method, the goodness of fit indices of BFNE-II indicates a fit that can be considered relatively sufficient. For BFNE-II, $\chi^{2} /$ $\mathrm{df}=3.62, \mathrm{TLI}=0.89, \mathrm{CFI}=0.91, \mathrm{SRMR}=0.05$ and RMSEA $=0.10$ (90\% CI=0.09-0.12). However, there was enough room to improve fitness. Due to the high load values of the scale items, the item removal method was not used. Correlated errors may result from items expressed similarly (40) or from items that are close to each other in the scale (41). Therefore, it was allowed to associate errors between items 5 and 6 , and between items 8 and 9, which are similarly expressed (have close meaning). The analysis was then performed again and it was observed that the error covariances significantly improved the model fit $\chi^{2} / \mathrm{df}=1.99$, TLI $=0.96$, CFI $=0.97$, $\mathrm{SRMR}=0.04$, RMSEA $=0.06(90 \% \mathrm{CI}=0.04-0.08)$. As a result, it was determined that the Turkish form of

Table 1: Descriptive statistics for BFNE-II and BFNE-S items

\begin{tabular}{lcc} 
& Mean & SD \\
\hline 1. I worry about what other people will think of me even when I know it doesn't make any difference. & 2.32 & 1.18 \\
2. It bothers me when people form an unfavourable impression of me. & 3.04 & 1.42 \\
3. I am frequently afraid of other people noticing my shortcomings. & 2.08 & 1.25 \\
4. I worry about what kind of impression I make on people. & 2.65 & 1.25 \\
5. I am afraid that others will not approve of me. & 1.99 & 1.12 \\
6. I am afraid that other people will find fault with me. & 1.81 & 1.12 \\
7.I am concerned about other people's opinions of me. & 2.08 & 1.12 \\
8. When I am talking to someone, I worry about what they may be thinking about me. & 2.23 & 1.26 \\
9.I am usually worried about what kind of impression I make. & 2.35 & 1.19 \\
10. If I know someone is judging me, it tends to bother me. & 2.99 & 1.41 \\
11. Sometimes I think I am too concerned with what other people think of me. & 2.31 & 1.42 \\
12. I often worry that I will say or do wrong things. & 2.42 & 1.35 \\
BFNE-II total & 28.27 & 11.05 \\
BFNE-S total & 17.51 & 7.54 \\
\hline
\end{tabular}

SD: Standard deviation 
BFNE-II corresponded to the original form, and the single-factor structure fits the data quite well. The factor loads of the items vary between 0.55 and 0.82 . As shown in Figure 1, all items of the scale were significantly loaded on a single factor with load values of 0.40 and above. It is seen that the standardized load values for all items are high and significant.

It is known that BFNE-S consists of a single-factor structure, which consists of eight items directly scored from BFNE and eight items of BFNE-II (the eight items mentioned in both scales are the same), and CFA was performed to test the single-factor structure. As a result of the initial analysis performed with the maximum likelihood estimation method, the goodness of fit indices of the BFNE-S indicates poor fit $\chi^{2} /$ $\mathrm{df}=6.16, \quad \mathrm{TLI}=0.86, \quad \mathrm{CFI}=0.90, \quad \mathrm{SRMR}=0.06$, RMSEA $=0.15$ (90\% CI=0.12-0.17), although there was enough room to improve the compliance. Due to the high load values of the scale items, no item removal method was used. The errors between items 3 and 4 and between items 5 and 6 were allowed to correlate among the scale items. It is also worth noting that items 3, 4, 5, and 6 in BFNE-S correspond to items 5, 6, 8 , and 9 in BFNE-II, respectively. Following these procedures, the analysis was performed again and the error covariances seem to significantly improve the model fit $\chi^{2} / \mathrm{df}=2.11$, TLI $=0.97, \mathrm{CFI}=0.98$, SRMR $=0.03$, RMSEA $=0.07$ (90\% CI=0.04-0.10). The factor loads of

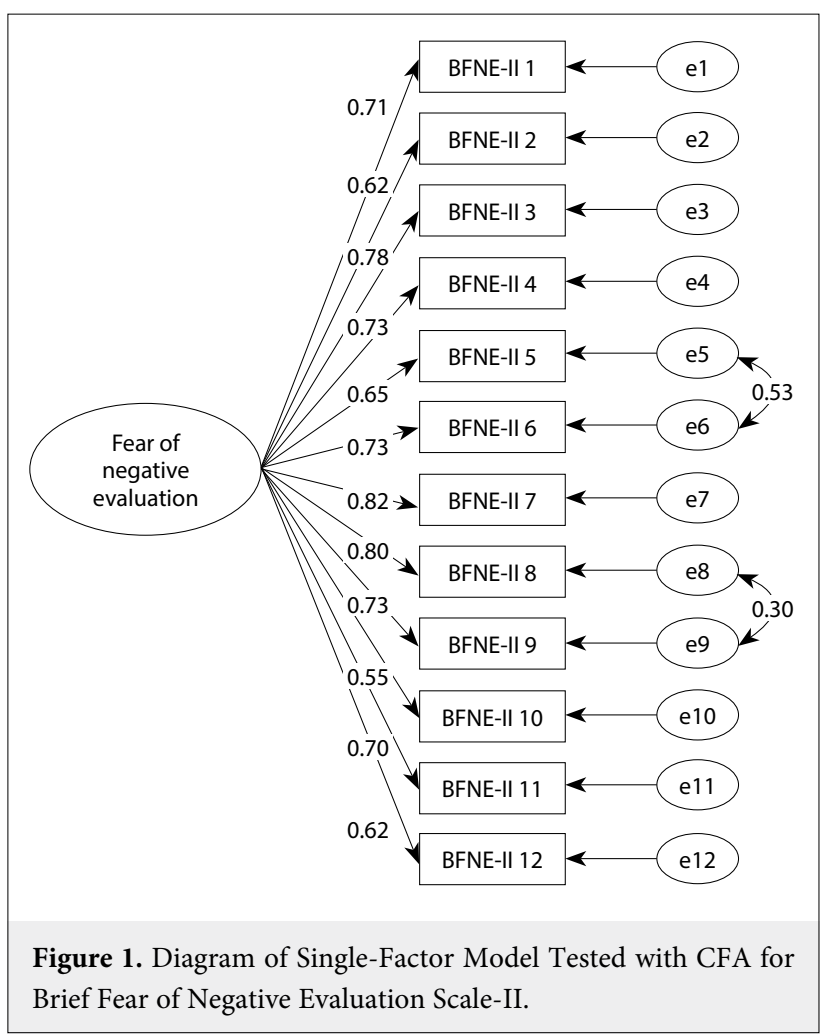

the items vary between 0.63 and 0.80 . As seen in Figure 2 , all items of the scale were significantly loaded on a single factor with load values 0.40 and above. It is observed that standardized load values for all items are high and significant.

\section{Concurrent Validity}

Correlations with FPES, RSES, LSAS, and its subdimensions (anxiety and avoidance) were calculated to test the concurrent validity of BFNE-II and BFNE-S. A statistically significant correlation $(\mathrm{p}<0.01)$ was found with all scales determined by BFNE-II and BFNE-S (Table 2). Examining in detail, there is a high level of correlation between BFNE-II and BFNE-S. BFNE-II has a moderate correlation with total LSAS and LSAS avoidance sub-dimension, while it has a low level of correlation with FPES, RSDS, and the anxiety sub-

Table 2: Pearson product-moments correlation coefficients of BFNE-II and BFNE-S with other associated scales

BFNE-II BFNE-S

\section{BFNE-II}

\begin{tabular}{llc} 
BFNE-S & $0.98^{*}$ & - \\
FPES & $0.26^{*}$ & $0.28^{*}$ \\
RSES & $0.29^{*}$ & $0.34^{*}$ \\
LSAS-Total & $0.32^{*}$ & $0.33^{*}$ \\
LSAS-Anxiety Subdimension & $0.23^{*}$ & $0.22^{*}$ \\
LSAS-Avoidance Subdimension & $0.33^{*}$ & $0.35^{*}$ \\
\hline
\end{tabular}

BFNE-II: Brief Fear of Negative Evaluation-II, BFNE-S: Brief Fear of Negative Evaluation Scale-Straightforward Items, FPES: Fear of Positive Evaluation Scale, RSES: Rosenberg Self-esteem Scale, LSAS: Liebowitz Social Anxiety Scale, ${ }^{*} \mathrm{p}<0.01$

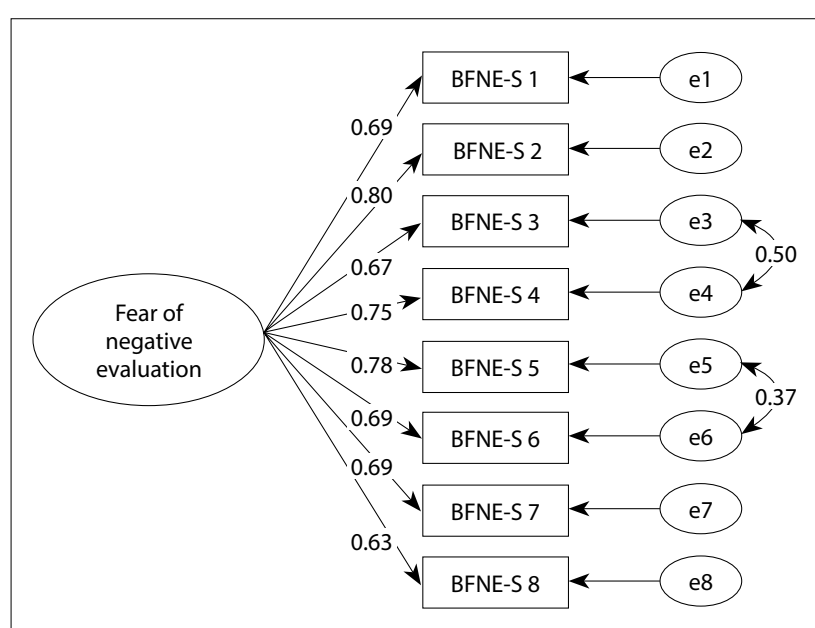

Figure 2. Diagram of Single-Factor Model Tested with CFA for Brief Fear of Negative Evaluation Scale- Straightforward Items. 
dimension of LSAS. BFNE-S has a moderate correlation with RSES, total LSAS, and LSAS avoidance subdimension, whereas it has a low-level correlation with FPES and LSAS anxiety subdimension. The determination of the effect sizes was based on the criteria proposed by Cohen (42).

\section{Findings Regarding Reliability}

The Cronbach's alpha value was calculated to test whether the BFNE-II is a reliable measurement tool. The Cronbach's alpha internal consistency coefficient of the scale consisting of a single factor structure was found to be 0.92 . The Cronbach's alpha coefficient obtained by the split-half method for BFNE-II was 0.87 for the first half and 0.82 for the second half. As a result of the analysis, the Spearman-Brown coefficient was found to be 0.94 and the Guttman test split-half coefficient also to be 0.94 . It was determined that the correlation coefficient between the scores obtained from the two forms was 0.89 . Findings obtained by the split-half test method show that BFNE-II is a reliable measurement tool. On the other hand, item-total correlations vary between 0.53 and 0.77 for the scale consisting of a single factor structure. Item-total correlation, common factor variance, and reliability values are shown in Table 3.

Following, Cronbach's alpha value was calculated to test whether BFNE-S is a reliable measurement tool. The Cronbach's alpha internal consistency coefficient of the scale consisting of a single factor structure was found to be 0.90 . The Cronbach's alpha coefficient obtained by the split-half method for BFNE-S was 0.80 for the first half and 0.81 for the second half. As a result of the analysis, the SpearmanBrown coefficient was 0.91, and the Guttman test split-half coefficient 0.91 . It was determined that the correlation coefficient between the scores obtained from the two forms was 0.84 . Findings obtained by the split-half test method show that BFNE-S was also a reliable measurement tool. On the other hand, itemtotal correlations vary between 0.60 and 0.75 for the scale consisting of a single factor structure. Item-total correlation, common factor variance and reliability values are shown in Table 4.

\section{Gender Differences}

As a result of the independent groups' t-test analysis performed with the BFNE-II version, it was found that women's levels of fear of negative evaluation $(\bar{X}=29.24$, $\mathrm{SD}=11.51)$ differed significantly and higher than men's levels of fear of negative evaluation $(\overline{\mathrm{X}}=25.30, \mathrm{SD}=8.94)$ $(\mathrm{t}[241]=2.42, \mathrm{p}<0.05)$. As a result of the independent groups' test analysis performed with the BFNE-S version, it was observed that women's level of fear of negative evaluation $(\overline{\mathrm{X}}=18.09, \mathrm{SD}=7.87)$, was again significantly higher than that of men $(\bar{X}=15.74$, $\mathrm{SD}=6.16)(\mathrm{t}[241]=2.12, \mathrm{p}<0.05)$.

\section{DISCUSSION}

Knowledge about the fear of negative evaluation and the psychometric properties of measurement tools is

Table 3: BFNE-II item-total correlations and reliability values

Cronbach's Alpha $=0.92$

\begin{tabular}{llcc} 
Items & Item-total correlations & $\begin{array}{c}\text { Communalities } \\
\text { Cronbach's Alpha value if the } \\
\text { item is deleted }\end{array}$ \\
\hline Item 1 & 0.68 & 0.51 & 0.913 \\
Item 2 & 0.59 & 0.43 & 0.917 \\
Item 3 & 0.74 & 0.61 & 0.910 \\
Item 4 & 0.70 & 0.53 & 0.912 \\
Item 5 & 0.64 & 0.59 & 0.915 \\
Item 6 & 0.72 & 0.68 & 0.912 \\
Item 7 & 0.77 & 0.63 & 0.910 \\
Item 8 & 0.76 & 0.66 & 0.909 \\
Item 9 & 0.71 & 0.59 & 0.912 \\
Item 10 & 0.53 & 0.32 & 0.920 \\
Item 11 & 0.68 & 0.48 & 0.913 \\
Item 12 & 0.59 & 0.38 & 0.917 \\
\hline
\end{tabular}

BFNE-II: Brief Fear of Negative Evaluation-II 
Table 4: BFNE-S item-total correlations and reliability values

Cronbach's Alpha $=0.90$

\begin{tabular}{lccc} 
Items & Item-total correlations & $\begin{array}{c}\text { Communalities } \\
\text { Cronbach's Alpha value if the } \\
\text { item is deleted }\end{array}$ & $\begin{array}{c}0.887 \\
\text { Item 1 }\end{array}$ \\
Item 2 & 0.63 & 0.43 & 0.877 \\
Item 3 & 0.73 & 0.59 & 0.884 \\
Item 4 & 0.66 & 0.58 & 0.878 \\
Item 5 & 0.73 & 0.66 & 0.875 \\
Item 6 & 0.75 & 0.62 & 0.881 \\
Item 7 & 0.69 & 0.55 & 0.885 \\
Item 8 & 0.66 & 0.45 & 0.890 \\
\hline
\end{tabular}

BFNE-S: Brief Fear of Negative Evaluation Scale-Straightforward Items

constantly increasing, and new suggestions are made for measurement tools. Adapting a more updated version of the scale seems important in the light of increasing information in the literature. Accordingly, this study has four objectives: (a) to verify the structure of BFNE-II and BFNE-S in the original form; (b) to examine its validity concurrently with the scales associated with social anxiety; (c) to test whether it is a reliable instrument of measurement; (d) and to determine its sensitivity in detecting gender differences found in previous studies (24).

The construct validity of the unitary model proposed for BFNE-II $(19,24)$ and BFNE-S $(8,17,43)$ in the Turkish sample was tested with CFA. The indices determined according to the value ranges determined for the goodness of fit statistics were examined. As a result, the $\chi^{2} / \mathrm{df}$ ratio, RMSEA, and SRMR values from the absolute fit indices and TLI and CFI values from incremental fit indices reflected a quite good fit for the single-factor model in parallel with the studies performed by the researchers for the BFNE-II and BFNE-S original forms. The single factor structure of BFNE-II and BFNE-S was verified according to CFA. In the light of this information, the hypotheses that the scales will consist of a single-factor structure as in the original form and that the fit values will be good and at an acceptable level have been confirmed. Examining the factor load, it was observed that the factor loads of all items were above the determined cut-off value for both scales. Therefore, factor loads of scale items were found to be sufficiently high and significant.

The concurrent validity method was also used within the scope of validity analysis. Statistically significant relationships were found between BFNE-II and BFNE-S and FPES, RSES, LSAS, and their sub-dimensions in the expected direction. Significantly positive correlations were found between BFNE-II and BFNE-S and FPES. This finding is consistent with other studies in the literature indicating a significant relationship between fear of positive evaluation and fear of negative evaluation $(25,44)$. Similar to the studies of Carleton et al. (24), a significant positive correlation was found between BFNEII and BFNE-S and RSES. As predicted, a stronger relationship was found between BFNE-II and LSAS compared to other scales. It is known that fear of negative evaluation comes to the fore as the main fear in social anxiety (4). A significant positive correlation was found between the anxiety and avoidance dimensions of LSAS and the BFNE-II and BFNE-S. Especially, it was determined in this study that the strongest relationship between BFNE-II and BFNE-S was with the avoidance sub-dimension. However, the significant but weak correlation between both scales and the anxiety subdimension of FPES and LSAS is remarkable.

When trying to determine why a correlation may be lower than expected, (a) low amount of variability in the data, (b) curvilinear relationships reported in some studies examining the relationships between anxiety and other variables, (c) sample's unique characteristics to affect its $r$ dimension, (d) sample selection's affecting the strength of relationships calculated with correlations, and (e) factors such as anxiety, fatigue and estimation, administrative factors, environmental factors, the uncertainty of questions, and the small number of questions stand out (45). In addition, the different sentence wording of the scales may cause poor correlation. The almost linear relationship between BFNE-II and BFNE-S, which has an almost similar sentence wording, and the poor correlation with FPES, whose sentence wording is different from both scales, suggest this fact. Apart from that, when examining things that are difficult to measure, such as the content 
of one's mental life, we can expect correlation coefficients to be lower. The varying but generally similar correlation levels of the same scales in different cultures and samples similar to those in our study seem to support this fact $(8,20,21,24)$. At the same time, it is thought that a low level of correlation coefficient does not mean that there is a lack of relationship more than a large correlation coefficient indicates a strong relationship. Moreover, having a poor correlation only means that the two variables have poor causation or have a poor relationship with each other, which does not mean they lack them. In conclusion, as a result of concurrent validity analysis, it was determined that BFNE-II and BFNE-S had significant relationships with the determined scales in accordance with the hypotheses of the research. These findings suggest that individuals with a high fear of negative evaluation also have high levels of social anxiety and low self-esteem. In particular, it is observed that individuals with high fear of negative evaluation have a higher avoidance level from the subdimensions of social anxiety.

Cronbach's alpha internal consistency reliability coefficient, item-total correlation, and values obtained by the split-half test were analyzed in the reliability analysis. Gliem and Gliem (46) suggest that a Cronbach's alpha value of 0.80 and above is a good indicator and the itemtotal correlation should be 0.40 and above. The Cronbach's alpha internal consistency reliability coefficient obtained as a result of the analysis shows that the scales have a very high level of internal consistency. The Cronbach's alpha internal consistency reliability coefficient obtained is at a similar level to the values obtained in the original form $(19,24)$. It is seen that the item-total correlations of the scales consisting of a singlefactor structure also have good values according to the determined cut-off point. Considering the split-half reliability of the test, it is seen that the obtained Cronbach's alpha coefficient has very good values for the first and the second half. When all the findings obtained as a result of the analysis are evaluated, it is considered that both variants of the scale are suitable for use in Turkish.

Reviewing the literature, it is revealed that the original form of BFNE-II (24) is a sensitive measurement tool for detecting gender differences. It was also determined in the Turkish version that BFNE-II is a sensitive measurement tool for determining gender differences. Again, it was concluded that the BFNE-S version was a sensitive measurement tool for determining gender differences.

Although the psychometric properties may seem to reveal a good framework at first, the study has some limitations. The first limitation concerns research sampling. The sample of the study consists of students in their emerging adulthood period who continue their university education. Considering the education levels and developmental stages of the participants, it may prevent the generalization of the findings to people of different ages and educational levels (such as those without university education). On the other hand, the unequal distribution of the sample by gender appears as another limitation; since the study does not have population-based assumptions, it is thought that this difference will not significantly affect the study results. However, in the context of collecting data from a nonclinical sample in the study, the generalizability of the data to clinical sampling is limited. Researching with different samples other than the university sample is important in future studies. In particular, studies with individuals diagnosed with SAD are needed. However, it is thought that including the clinical group as well as the non-clinical group in the research will allow comparison of the two groups and provide more comprehensive information. In addition, it is another important point that the men/women ratio should be more balanced to minimize the possible effects of gender on results in future studies. Finally, in this study, two multiple regression analyzes can be performed on the total scores of the variables used for concurrent validity (FPES, LSAS, and RSES), along with demographic variables (age and gender), on the BFNEII and BFNE-S total scores. Since there are significant differences for both scales for gender in the analyzes conducted in this and the previous studies (24), the results obtained here may provide significant contributions to the relevant literature.

In conclusion, the findings indicate that BFNE-II and BFNE-S are reliable and valid measurement tools for Turkish use. Therefore, BFNE-II and BFNE-S appear to be useful measurement tools in determining the level of fear of negative evaluation, one of the main components of social anxiety. In addition, it is known that fear negative evaluation in cognitive models of social anxiety $(2,3)$ is one of the important factors in the continuation of the disorder. Therefore, BFNE-II and BFNE-S are considered useful measurement tools in future SAD studies. While the eight-item BFNE-S can be preferred due to its short-term applicability and less sensitivity in gender differences among the versions of BFNE-II and BFNE-S, which have similar levels of adjustment values, the twelve-item BFNE-II can be preferred against the loss of sensitivity that may arise in fear of negative evaluation. 


\begin{tabular}{|c|c|c|}
\hline \multicolumn{2}{|c|}{ Contribution Categories } & \multirow{2}{*}{\begin{tabular}{|l|} 
Author Initials \\
B.G.G., O.Y.A. \\
\end{tabular}} \\
\hline \multirow{3}{*}{ Category 1} & Concept/Design & \\
\hline & Data acquisition & B.G.G., O.Y.A. \\
\hline & Data analysis/Interpretation & B.G.G. \\
\hline \multirow{2}{*}{ Category 2} & Drafting manuscript & B.G.G. \\
\hline & Critical revision of manuscript & O.Y.A. \\
\hline Category 3 & Final approval and accountability & B.G.G., O.Y.A. \\
\hline \multirow{2}{*}{ Other } & Technical or material support & N/A \\
\hline & Supervision & N/A \\
\hline
\end{tabular}

Ethics Committee Approval: This research was approved by the Ankara Yildirim Beyazit University Ethics Committee (Number: 2018-214 Date: 28.06.2018/14).

Informed Consent: Informed consent was obtained from all participants.

Peer-review: Externally peer-reviewed.

Conflict of Interest: The authors declared no conflict of interest.

Financial Disclosure: All financial expenses of the study were covered by the authors.

\section{REFERENCES}

1. American Psychiatric Association. Diagnostic and statistical manual of mental disorders. Fifth Ed. Arlington: American Psychiatric Association, 2013. [CrossRef]

2. Clark DM, Wells A. A cognitive model of social phobia. In Liebowitz M, Heimberg RG (editors). Social phobia: Diagnosis, assessment, and treatment. New York: Guilford Press, 1995, 69-93.

3. Rapee RM, Heimberg RG. A cognitive-behavioral model of anxiety in social phobia. Behav Res Ther 1997; 35:741-756.

4. Beck AT, Emery G, Greenberg RL. Anxiety disorders and phobias: A Cognitive Approach. New York: Basic Books, 1985, 146-164.

5. Coles ME, Turk CL, Heimberg RG, Fresco DM. Effects of varying levels of anxiety within social situations: relationship to memory perspective and attributions in social phobia. Behav Res Ther 2001; 39:651-665. [CrossRef]

6. Hackmann A, Surawy C, Clark DM. Seeing yourself through others' eyes: A study of spontaneously occurring images in social phobia. Behav Cogn Psychother 1998; 26:3-12. [CrossRef]

7. Wells A, Papageorgiou C. Social phobia: Effects of external attention on anxiety, negative beliefs, and perspective taking. Behav Ther 1998; 29:357-370. [CrossRef]

8. Weeks JW, Heimberg RG, Fresco DM, Hart TA, Turk CL, Schneier FR, et al. Empirical validation and psychometric evaluation of the Brief Fear of Negative Evaluation Scale in patients with social anxiety disorder. Psychol Assess 2005; 17:179-190. [CrossRef]

9. Watson D, Friend R. Measurement of social-evaluative anxiety. J Consult Clin Psychol 1969; 33:448-457. [CrossRef]

10. Stopa L, Clark DM. Social phobia: Comments on the viability and validity of an analogue research strategy and British norms for the fear of negative evaluation questionnaire. Behav Cogn Psychother 2001; 29:423-430. [CrossRef]

11. Erkan Z, Cam S, Gucray S. Adaptation, validity and reliability study of Social Avoidance and Anxiety and Fear of Negative Evaluation Scales. Cukurova University Faculty of Education Journal 2003; 2:112-117.

12. Leary MR. A brief version of the Fear of Negative Evaluation Scale. Pers Soc Psychol Bull 1983; 9:371-375. [CrossRef]

13. Koydemir S, Demir A. Psychometric properties of the brief version of the Fear of Negative Evaluation Scale in a Turkish sample. Psychol Rep 2007; 100:883-893. [CrossRef]

14. Bilge F, Kelecioglu H. Psychometric properties of the Brief Fear of Negative Evaluation Scale: Turkish Form. Eurasian J Educ Res 2008; 32:21-38.

15. Cetin B, Dogan T, Sapmaz F. Turkish adaptation of the Brief Fear of Negative Evaluation Scale Form: a validity and reliability study. Education and Science 2010; 35:205-216.

16. Turner SM, McCanna M, Beidel DC. Validity of the Social Avoidance and Distress and Fear of Negative Evaluation Scales. Behav Res Ther 1987; 25:113-115. [CrossRef]

17. Rodebaugh TL, Woods CM, Thissen DM, Heimberg RG, Chambless DL, Rapee RM. More information from fewer questions: the factor structure and item properties of the original and Brief Fear of Negative Evaluation Scale. Psychol Assess 2004; 16:169-181. [CrossRef]

18. Oei TP, Kenna D, Evans L. The reliability, validity and utility of the SAD and FNE scales for anxiety disorder patients. Pers Individ Dif 1991; 12:111-116. [CrossRef]

19. Carleton RN, McCreary DR, Norton PJ, Asmundson GJ. Brief Fear of Negative Evaluation Scale-Revised. Depress Anxiety 2006; 23:297-303. [CrossRef]

20. Pitarch MJ. Brief version of the Fear of Negative Evaluation Scale - Straightforward Items (BFNE-S): psychometric properties in a Spanish population. Span J Psychol 2010; 13:981-989. [CrossRef]

21. Tavoli A, Melyani M, Bakhtiari M, Ghaedi GH, Montazeri A. The Brief Fear of Negative Evaluation Scale (BFNE): translation and validation study of the Iranian version. BMC Psychiatry 2009; 9:42.

22. Wei J, Zhang C, Li Y, Xue S, Zhang J. Psychometric properties of the Chinese version of the Brief Fear of Negative Evaluation Scale (BFNE) and the BFNE-straightforward for middle school students. PLoS One 2015; 10:e0115948. [CrossRef]

23. Costello $\mathrm{AB}$, Osborne J. Best practices in exploratory factor analysis: Four recommendations for getting the most from your analysis. Pract Assess Res Eval 2005; 10:1-9.

24. Carleton RN, Collimore KC, Asmundson GJ. Social anxiety and fear of negative evaluation: construct validity of the BFNE-II. J Anxiety Disord 2007; 21:131-141. [CrossRef]

25. Weeks JW, Heimberg RG, Rodebaugh TL. The Fear of Positive Evaluation Scale: assessing a proposed cognitive component of social anxiety. J Anxiety Disord 2008; 22:44-55. [CrossRef]

26. Dogan T, Totan T. Validity and reliability study of the Turkish form of the Fear of Positive Evaluation Scale. Academic Perspective 2010; 22:1-20. 
27. Liebowitz MR. Social phobia. Mod Probl Pharmacopsychiatry 1987; 22:141-173. [CrossRef]

28. Heimberg RG, Horner KJ, Juster HR, Safren SA, Brown EJ, Schneier FR, et al. Psychometric properties of the Liebowitz Social Anxiety Scale. Psychol Med 1999; 29:199-212. [CrossRef]

29. Soykan C, Ozguven HD, Gencoz T. Liebowitz Social Anxiety Scale: the Turkish version. Psychol Rep 2003; 93:1059-1069.

30. Rosenberg M. Society and the adolescent self-image. Princeton: Princeton University Press, 1965, 16-36. [CrossRef]

31. Cuhadaroğlu F. Self-esteem in adolescents. Unpublished dissertation. Ankara: Hacettepe University, 1986.

32. Hambleton RK, Patsula L. Increasing the validity of adapted tests: Myths to be avoided and guidelines for improving test adaptation practices. Journal of Applied Testing Technology 1999; 1:1-13.

33. George D, Mallery M. IBM SPSS statistics 23 step by step: a simple guide and reference. 14th ed., New York: Routledge, 2016, 112-120.

34. Barrett P. Structural equation modelling: Adjudging model fit. Pers Individ Dif 2007; 42:815-824. [CrossRef]

35. Schreiber JB, Nora A, Stage FK, Barlow EA, King J. Reporting structural equation modeling and confirmatory factor analysis results: A review. J Educ Res 2006; 99:323-338. [CrossRef]

36. Ullman JB. Structural equation modeling: In Tabachnick BG, Fidell LS (editors). Using multivariate statistics. Sixth Ed. Harlow: Pearson Education Limited, 2014, 731-836.

37. Hu LT, Bentler PM. Cutoff criteria for fit indexes in covariance structure analysis: Conventional criteria versus new alternatives. Struct Equ Modeling 1999; 6:1-55. [CrossRef]
38. Browne MW, Cudeck R. Alternative ways of assessing model fit. Sociol Methods Res 1992; 21:230-258. [CrossRef]

39. Hu LT, Bentler PM. Evaluating model fit. Structural equation modeling: In Hoyle RH (editor). Concepts, issues, and applications. London: Sage, 1995, 76-99.

40. Brown TA. Confirmatory factor analysis for applied research. New York: Guilford Publications, 2015, 157-162.

41. Bollen, K, Lennox R. Conventional wisdom on measurement: A structural equation perspective. Psychol Bull 1991; 110:305314. [CrossRef]

42. Cohen J. Statistical power analysis for the behavioral sciences. Second ed. Hillsdale: Erlbaum, 1988, 75-83.

43. Carleton RN, Collimore KC, McCabe RE, Antony MM. Addressing revisions to the brief fear of negative evaluation scale: measuring fear of negative evaluation across anxiety and mood disorders. J Anxiety Disord 2011; 25:822-828. [CrossRef]

44. Fergus TA, Valentiner DP, McGrath PB, Stephenson K, Gier S, Jencius S. The Fear of Positive Evaluation Scale: psychometric properties in a clinical sample. J Anxiety Disord 2009; 23:11771183. [CrossRef]

45. Goodwin LD, Leech, NL. Understanding correlation: Factors that affect the size of r. J Exp Educ 2006; 74:249-266. [CrossRef]

46. Gliem JA, Gliem RR. Calculating, interpreting, and reporting Cronbach's alpha reliability coefficient for Likert-type scales. Presented at the Midwest Research-to-Practice Conference in Adult, Continuing, and Community Education. Columbus: The Ohio State University, 2003, 82-88. 